\title{
The use of anti-tuberculosis therapy for latent TB infection
}

This article was published in the following Dove Press journal:

Infection and Drug Resistance

20 July 2010

Number of times this article has been viewed

\section{Justin T Denholm ${ }^{1,2}$}

Emma S McBryde ${ }^{1,2}$

'Victorian Infectious Diseases Service, Royal Melbourne Hospital, Melbourne, Victoria, Australia; ${ }^{2}$ Department of Medicine, (RMH/WH), University of Melbourne, Parkville, Victoria, Australia
Correspondence: Justin T Denholm Victorian Infectious Diseases Service, Royal Melbourne Hospital, 9N, Grattan Street, Parkville, Victoria, Australia 3050 Tel +6I 393427000

Fax +6I 393427277

Email justin.denholm@mh.org.au
Abstract: Tuberculosis infection is of global public health significance, with millions of incident cases each year. Many cases, particularly in low-prevalence settings, result from the reactivation of latent tuberculosis infection (LTBI); potentially acquired years prior to active disease. Up to one-third of the world's population has been infected with LTBI, and so may be at risk for future active TB disease. A variety of antituberculosis medications and treatment regimens have now been evaluated in the management of LTBI, with the aim of eradicating tuberculosis bacilli and reducing the likelihood of subsequent reactivation disease. This article reviews LTBI therapies and their use in clinical contexts, and considers future directions for individual and population-based strategies in LTBI management.

Keywords: tuberculosis, latent tuberculosis, isoniazid, rifampicin, tuberculin skin test, interferon-gamma release assay

\section{Introduction}

It is estimated that active tuberculosis (TB) infection results in 9-10 million incident cases and around 1.8 million deaths annually. ${ }^{1}$ Estimates of the prevalence of latent tuberculosis infection (LTBI) are considerably less precise, but it is commonly suggested that up to one-third of the world's population may be affected and at risk of subsequent reactivation. LTBI is therefore a condition of considerable importance. This is particularly so in settings with a low prevalence of active TB, where the majority of infections relate to reactivation of latent disease.

LTBI is by definition an asymptomatic condition. There is no gold standard test for LTBI, and establishing a diagnosis may involve elements of medical history, chest $\mathrm{X}$-ray, tuberculin skin test (TST), or interferon-gamma release assay (IGRA). Uncertainty related to the diagnosis makes rational public health use of LTBI treatment difficult, and better tools for estimating population prevalence are required. Although there continues to be uncertainty regarding the precise likelihood of reactivation in individuals, patients with LTBI may experience reactivation decades after exposure, with an often quoted summary estimate of $10 \%$ lifetime risk of progressing to active infection. ${ }^{2}$ A variety of antituberculosis medications and treatment regimens have been evaluated in the management of LTBI, with the aim of eradicating tuberculosis bacilli and reducing the likelihood of subsequent reactivation disease.

This article will review LTBI therapies and their use in clinical contexts, and will consider future directions for individual and population-based strategies in LTBI management. 


\section{General principles of LTBI therapy}

The antimicrobial agents used for the treatment of active tuberculosis are also those used and investigated for LTBI. However, not all medications effective against active tuberculosis are necessarily appropriate for the treatment of LTBI. Medications must be active against the latent phase of M. tuberculosis and be sufficiently well-tolerated for treatment of an asymptomatic condition. Preferably, they should also require little regular monitoring, sterilize mycobacteria quickly and have a high threshold to the development of microbial resistance.

Treatment of LTBI at present requires extended courses of effective antituberculosis therapy. Although strategies are being investigated to shorten the duration of these treatment regimens, it is expected that the long latent periods and slow replicative cycle of $M$. tuberculosis will mean that treatment with existing agents will continue to be relatively lengthy for the foreseeable future. Accordingly, all regimens for the treatment of LTBI currently in use require months of daily anti-tuberculosis medications. Lengthy courses of therapy in asymptomatic patients are typically associated with high rates of poor adherence and loss to follow-up, with resultant decrease in efficacy of LTBI treatment programs. ${ }^{3}$ Strategies to improve adherence with therapy have included educational programs, support from pharmacists and other allied health care providers, medication alarms and reminder systems; however the establishment of effective short-course regimens are ultimately likely to provide the most benefit in this setting. ${ }^{46}$

In an attempt to improve compliance, intermittent dosing strategies for various LTBI regimens have been investigated, such as twice-weekly isoniazid instead of daily administration. These approaches will be reviewed in more detail below; however as a general principle, non-compliance is more likely to impact outcome if the regimen is intermittent rather than daily. Accordingly, it is recommended that all non-daily dosing schedules be administered by directly observed therapy (DOT) to ensure compliance.

Typically, antimicrobial agents for LTBI have been prescribed as monotherapy, most commonly with isoniazid or rifampicin (see below). In the setting of active tuberculosis, the use of single-medication therapy clearly leads to the development of antimicrobial resistance. For instance, when isoniazid alone was used for disease treatment, $71 \%$ of patients developed resistance after three months. ${ }^{7}$ Rather than being caused by the induction of resistance in individual organisms, this is believed to be due to the selection of small numbers of naturally resistant $M$. tuberculosis organisms present at the time of treatment. However, the low number of bacilli present in latent tuberculosis infections means that the presence of any such naturally resistant organisms is uncommon, and monotherapy is generally effective. In situations where there is a high probability of drug-resistant organisms present, such a principle does not remain reliable, and alternative strategies may be required (see below).

As LTBI itself is an asymptomatic condition, treatment is given based on the risk of future reactivation. The decision to treat, therefore, involves a weighing of the risk of therapy against benefit from reduction in reactivation for the individual patient. Such decisions will necessarily be influenced by factors that either increase risk of reactivation (such as recent contact with active tuberculosis or the impending introduction of immunosuppressive medication) or risk of serious adverse effects of therapy (such as advanced age or pre-existing hepatotoxicity). In most settings, these factors are sufficiently varied that treatment decisions must be individually considered and made, rather than being applicable to broader populations outside of limited settings.

Overall, while the effectiveness of treatment for LTBI is clear from large studies such as those described here, it is important to recognize that there remains no test or method for determining whether treatment have been effective in the individual patient. Patients therefore should be counseled that treatment success cannot be guaranteed, and symptoms consistent with active tuberculosis should be investigated appropriately even following completion of LTBI treatment.

\section{LTBI in people with HIV}

Co-infection with HIV and tuberculosis is recognized as a significant issue worldwide. Although much of this awareness relates to the problems associated with active infection, the diagnosis and treatment of LTBI in people with HIV presents additional difficulties. Although HIV-positive people may be no more likely to be exposed to tuberculosis, once exposed they have considerably increased risk of progression to active disease, whether primary or secondary. ${ }^{8}$ Active tuberculosis is less likely to present in classic pulmonary forms in people co-infected with HIV, and it may be more difficult to exclude active infection than in those without HIV. ${ }^{9-11}$ This is problematic for the appropriate management of LTBI, as initiating therapy in the setting of unrecognized active infection may lead to treatment failure and the development of anti-tuberculosis drug resistance. For instance, one study of HIV-infected subjects in Cote d'Ivoire found that 1.9\% of 
enrolled subjects had active tuberculosis at baseline, despite clinical screening intended to exclude such patients. ${ }^{12}$

Interactions between antiretroviral medications and treatment for LTBI may also be an important issue in both safety and effectiveness, and is dealt with in the relevant sections below.

\section{Regimens for treatment of LTBI Isoniazid}

By far the most established and widely used medication for the treatment of LTBI is isoniazid. First identified in the early 1950 's, isoniazid has multiple effects on mycobacterial metabolism, including inhibition of mycolic acid synthesis. ${ }^{13,14} \mathrm{Sev}-$ eral dosing strategies are commonly employed, with evidence for both daily self-administered therapy and supervised administration (DOT) twice weekly in some settings. A summary of current dosing recommendations from the US Centers for Disease Control and Prevention can be seen in Table 1.

Initial human trials of isoniazid preventative therapy established that prolonged therapy with isoniazid was effective in reducing subsequent active tuberculosis infections. In one of the earliest studies conducted, 800 Kenyan tuberculosis contacts were randomized to either receive 12 months of isoniazid or placebo. ${ }^{15}$ A $90 \%$ reduction in active TB diagnosis

Table I Dosing regimens for tuberculosis

\begin{tabular}{|c|c|c|c|}
\hline \multirow[t]{2}{*}{ Drug/dose } & \multirow{2}{*}{$\begin{array}{l}\text { Frequencyl } \\
\text { duration }\end{array}$} & \multicolumn{2}{|c|}{ Rating (evidence) } \\
\hline & & $\begin{array}{l}\text { HIV } \\
\text { negative }\end{array}$ & $\begin{array}{l}\text { HIV } \\
\text { positive }\end{array}$ \\
\hline \multicolumn{4}{|l|}{ Preferred Regimen } \\
\hline Isoniazid & Daily $\times$ & A (II) & A (II) \\
\hline Adult: 5 mg/kg & 9 months & & \\
\hline \multicolumn{4}{|l|}{ Children: $10-20 \mathrm{mg} / \mathrm{kg}$} \\
\hline \multicolumn{4}{|l|}{ Maximum dose $300 \mathrm{mg}$} \\
\hline \multicolumn{4}{|l|}{ Alternate Regimens } \\
\hline Isoniazid & Twice weekly $\times$ & B (II) & B (II) \\
\hline Adult: 15 mg/kg & 9 months & & \\
\hline \multicolumn{4}{|l|}{ Children: $20-40$ mg/kg } \\
\hline \multicolumn{4}{|l|}{ Maximum dose $900 \mathrm{mg}$} \\
\hline Isoniazid & Daily $\times$ & $B(I)$ & $C(I)$ \\
\hline Adults: 5 mg/kg & 6 months & & \\
\hline \multicolumn{4}{|l|}{ Maximum dose $300 \mathrm{mg}$} \\
\hline Isoniazid & Twice weekly $\times$ & B (II) & $C(I)$ \\
\hline Adults: $15 \mathrm{mg} / \mathrm{kg}$ & 6 months & & \\
\hline \multicolumn{4}{|l|}{ Maximum dose $900 \mathrm{mg}$} \\
\hline Rifampin & Daily $x$ & B (II) & B (II) \\
\hline Adults: $10 \mathrm{mg} / \mathrm{kg}$ & 4 months & & \\
\hline \multicolumn{4}{|l|}{ Children: $10-20$ mg/kg } \\
\hline Maximum dose 600 mg & & & \\
\hline
\end{tabular}

Notes: Reproduced from: Centers for Disease Control and Prevention. Guide for Primary Health Care Providers: Targeted tuberculin testing and treatment of latenttuberculosis infection. CDC, 2005. Available at www.cdc.gov/tb/publications/ LTBI/intro.htm was observed in the isoniazid intervention arm, with 17 versus 2 patients developing active infections after 1 year. Studies investigating the use of longer courses found that no significant additive benefit accrued beyond 12 months of therapy. ${ }^{16} \mathrm{Sub}-$ sequent studies investigated the potential of shorter courses of isoniazid therapy, such as the large International Union Against Tuberculosis (IUAT) trial. ${ }^{17}$ This international multicenter study randomized 28,000 subjects with fibrotic pulmonary lesions to receive 3,6 , or 12 months of isoniazid treatment (previously known as isoniazid preventive therapy, or IPT) or placebo, with follow-up continued for 5 years. While 12 months of isoniazid therapy prevented the largest number cases of active tuberculosis ( $75 \%$ reduction from placebo; compared to $21 \%$ and $65 \%$ with 3 and 6 months, respectively), it was also associated with a higher rate of serious hepatotoxicty. The study concluded that 6 months was the optimal duration, as it prevented the greatest number of tuberculosis infections per episode of hepatitis caused. Subsequently, a re-analysis of these results suggested that amongst patients compliant with treatment, this reduction was $69 \%$ for 6 months of isoniazid and $93 \%$ for 12 months. ${ }^{18}$ This analysis also reviewed additional studies and recommended that the optimal duration of treatment was likely to be ' 9 or 10 months', although no specific trial data was available for this duration of therapy. Nonetheless this recommendation has become widely adopted, particularly in the United States where it forms the basis for national guidelines recommending this duration.

\section{Isoniazid therapy in people with HIV}

With widespread co-infection of HIV and TB, particularly in Sub-Saharan Africa, the effectiveness of isoniazid preventative therapy in various clinical settings with high HIV prevalence has also been explored. One placebo-controlled trial of 12 months of isoniazid in HIV-infected children has been conducted, in which HIV-infected infants in a high prevalence TB area were enrolled. ${ }^{19}$ Children were predominantly not receiving highly active anti-retroviral therapy. This study was stopped early after an interim report found a significant reduction in tuberculosis diagnosis and mortality in children receiving isoniazid (8\% vs 16\% after a mean follow-up of 5.7 months). A follow up report on this study population suggested a very high compliance with prescribed therapy $(>90 \%) .^{20}$

The long-term benefits of routine LTBI treatment in high prevalence settings are unknown, and further studies will be required to determine optimal strategies, including appropriate settings, duration of use and impact on drug-resistance in subsequent active tuberculosis infections. In HIV-infected 
adults with LTBI, meta-analysis of published studies has confirmed that isoniazid therapy is effective in preventing progression to active infection..$^{21}$ This analysis of seven studies, including 4529 subjects, suggested a relative risk (RR) of 0.4 (95\% confidence interval [CI]: $0.24-0.65)$ in those with a positive TST, while a nonsignificant reduction (0.84, 95\% CI: $0.54-1.30)$ was found in those with negative baseline testing. However, other studies have shown that unselected HIV-positive populations may also benefit from LTBI therapy in areas with sufficiently high TB prevalence. ${ }^{22}$ Current guidelines recommend that the HIV-positive people follow the same treatment protocols for therapy as HIVnegative people diagnosed with LTBI, with a 9-month course as optimal duration. ${ }^{3}$

\section{Isoniazid treatment in children and adolescents}

Isoniazid is regarded as safe in children, and is widely used for the treatment of LTBI from infancy. There is an increased rate of progression to active TB in children aged less than 5 years old, and LTBI therapy in this group is highly effective, perhaps more so than in adults. In households with active tuberculosis infection, some studies have estimated that up to $30 \%-40 \%$ of children under 15 years may have LTBI. ${ }^{23,24}$ Several large early trials have demonstrated that the risk of progression to active disease may be reduced by up to $90 \%$ with 12 -month courses of therapy. ${ }^{25}$ Accordingly, WHO guidelines recommend that all TB-exposed children in whom active infection has been excluded receive a course of treatment for LTBI, however this is not routinely provided in many developing world settings. ${ }^{26,27}$

\section{Isoniazid treatment in pregnancy and lactation}

Isoniazid does not have teratogenic effects in humans, and has been widely used in regimens for the treatment of active infections in pregnancy. ${ }^{28,29}$ Serious hepatotoxity has been reported during isoniazid use in pregnancy, and one retrospective analysis of cases suggested a 2.5 -fold (but not statistically significant) increase in hepatotoxicty in this setting. ${ }^{30,31}$ Treatment for LTBI in pregnancy is sometimes deferred until after delivery for this reason, as pregnancy per se does not influence the risk of TB reactivation. ${ }^{32}$ However, an evaluation of various strategies has concluded that treating LTBI during pregnancy with isoniazid would be cost effective and improve overall outcomes, with increased hepatotoxicty more than offset by decreased tuberculosis infections. ${ }^{33}$ Guidelines generally support treatment of LTBI in pregnancy with isoniazid as the preferred option where there is high risk of reactivation, with close monitoring of liver function tests recommended. ${ }^{3}$
Small amounts of active drug are present in breast milk when isoniazid is used in lactating women, in insufficient amounts for the treatment of LTBI in infants. ${ }^{3}$ No adverse effects on infants have been reported, however it is recommended that pyridoxine be given to breastfeeding infants when isoniazid is used in this setting. ${ }^{34}$

\section{Adverse effects and tolerability}

A number of large studies and reviews of isoniazid monotherapy have concluded that the incidence of serious hepatitis (ALT > 5 ULN) in young, previously well patients is between $0.1 \%-0.56 \%$, a figure adopted by the American Thoracic Society for formulation of guidelines and recommendations. ${ }^{35-37}$ However, when routine liver function testing is performed throughout isoniazid therapy, discontinuation of medication occurs in around $4 \%-10 \%$ of patients due to abnormal results. ${ }^{38-40}$ In a recent randomized trial, for instance, discontinuation due to serious hepatotoxicity was reported in $3.8 \%$ of patients..$^{40}$ Patients in this study included those at higher risk of side effects, including abnormal baseline LFT and those aged $>35$. The discordance between these liver function test results and clinical syndromes seen in various studies has led to recommendations against routine liver function testing during LTBI treatment in low risk patients, particularly those aged $<35$ with normal baseline liver function. ${ }^{41}$

Peripheral neuropathy has been recognised in association with isoniazid preventive therapy since its inception. ${ }^{42}$ Isoniazid leads to neuropathy by competitively inhibiting the metabolic activity of pyridoxine, an activity that can be overcome in at-risk patients through the co-administration of pyridoxine supplementation. A genetic basis for this toxicity has been identified in variable acetylation of isoniazid between individuals, although malnutrition may also play a role in increasing neuropathy risk. ${ }^{43}$ Co-prescription of other neurotoxic agents such as stavudine (D4T) or didanosine should be avoided due to increased rates of peripheral neuropathy ${ }^{44}$ Unlike the treatment of active tuberculosis, treatment regimens for LTBI are not usually supplemented by pyridoxine, but it may be considered in high-risk groups, including those with pre-existing neuropathy or additional risk factors for its development (eg, HIV, diabetes, malnutrition or use of other neurotoxic medications). ${ }^{3}$

\section{Isoniazid resistance}

High prevalence tuberculosis regions worldwide have reported increasing rates of isoniazid resistance, with the resulting potential for decreasing effectiveness of isoniazid-based regimens for LTBI. ${ }^{45}$ South African serial surveys of 
antimicrobial sensitivity found an increase in isoniazid resistance from $6.9 \%$ to $12.4 \%$ in pediatric TB isolates between 1994-2005, a significant development in a treatment naïve cohort. ${ }^{46}$ In regions such as Uzbekistan and Azerbaijan, with a high proportion of treatment failure and MDR-TB transmission, reported isoniazid-resistance is as high as $40 \%-49 \% .{ }^{47}$ Clearly in settings such as these, the effectiveness of isoniazid treatment will be considerably reduced, and alternative strategies for LTBI management are required.

\section{Rifampicin (rifampin)}

Rifampicin acts by inhibiting bacterial DNA polymerase, and is currently recommended as second-line therapy for LTBI, or for use where the index case is known to be infected with isoniazid-resistant tuberculosis. ${ }^{3}$

Rifampicin has been regarded as an attractive option for LTBI treatment due to the possibility of shortening duration of therapy, as was seen when the medication was introduced into treatment of active tuberculosis. The only randomized trial of rifampicin monotherapy for LTBI compared 3 months of rifampicin with 6 months of isoniazid therapy in patients with silicosis, and concluded that they were equivalent in effectiveness. ${ }^{48} \mathrm{~A}$ further retrospective study including 49 people exposed to isoniazid-resistant tuberculosis found that none progressed to active disease a mean 26 months after LTBI therapy with rifampicin monotherapy; however duration of therapy was nonstandardized and often prolonged, with a mean duration of more than 6 months. ${ }^{49}$ In light of the probable increased effectiveness of 9 month courses of isoniazid over 6 month courses, subsequent adult studies have favored treatment regimens longer than 3 months, usually 4-month courses of rifampicin. ${ }^{50}$ No study, however, has directly compared the effectiveness of 4 months of rifampicin against 9 months of isoniazid for the prevention of active tuberculosis; a key requirement if this regimen is to be used more widely in the future.

\section{Rifampicin therapy in people with HIV}

Rifampicin monotherapy for LTBI has not been studied in HIV-positive cohorts, with some authorities citing concerns about unrecognized active disease and the potential for development of rifampicin resistance. ${ }^{51}$ Rifampicin also interacts with a number of common antiretroviral medications such as efavirenz, and in particular rifampicin-containing regimens should be avoided in patients being treated with protease inhibitors (PI), as enzyme induction may lead to sub-therapeutic levels of PI and increased rifampicin toxicity. ${ }^{52}$ Pharmacokinetic studies have suggested that PI boosted with ritonavir may still achieve acceptable serum levels however there has been no evaluation of LTBI therapy in this context, and rifampicin monotherapy is not recommended. ${ }^{53}$

\section{Rifampicin in children and adolescents}

Rifampicin is recommended as an alternative to 9 months of isoniazid for children. Typically, 4-6 month durations are recommended, with no studies directly comparing the two regimens. In one series, 157 adolescent patients exposed to isoniazid-resistant index cases of tuberculosis were treated with a 6-month course of rifampicin, with no progression to active disease observed over a 2 -year follow-up period. ${ }^{54}$

\section{Rifampicin in pregnancy and lactation}

There are no controlled studies of rifampicin in pregnancy, and retrospective reviews are divided regarding any increased risk of congenital malformations. ${ }^{32}$ Use of rifampicin for latent tuberculosis is not recommended in pregnancy, although it has been used widely in the treatment of active tuberculosis in this context. ${ }^{3}$ Rifampicin is also present in breast milk, at low concentrations that are considered safe for infants, but nontherapeutic. ${ }^{55}$

\section{Adverse effects and tolerability}

The adverse effects of rifampicin have been compared with isoniazid in a recent international randomized trial. ${ }^{40}$ Patients diagnosed with LTBI $(n=847)$ were randomized to receive either 4-month courses of rifampicin or 9 months of isoniazid, with early cessation of enrolment due to decreased serious adverse events in the rifampicin-receiving arm. Patients prescribed isoniazid were more likely to have serious adverse events overall (4.0\% vs $1.7 \%$ ), with the bulk of adverse events relating to hepatotoxicity. Hepatotoxicity occurred in $0.7 \%$ of patients receiving rifampicin and in $3.8 \%$ of patients receiving isoniazid. While the number of patients found to have developed isoniazid hepatotoxicity in this study is high compared to previous investigations, this likely reflects a greater-risk patient group and represents a fair assessment of the relative toxicity of the two regimens.

\section{Rifampicin and pyrazinamide}

Initial studies of 2-month rifampicin and pyrazinamide (2RZ) regimens evaluated its effectiveness predominantly in settings with a high prevalence of both HIV/AIDS and tuberculosis. ${ }^{56}$ The first of these studies suggested that $2 \mathrm{RZ}$ moderately reduced the incidence of subsequent TB infection (RR 0.58 over 1 year; 95\% CI: 0.35-0.95) when compared 
with placebo; reduction is somewhat less effective than a 6 month course of isoniazid. This effect waned quickly after treatment cessation, likely reflecting TB re-infection in a high prevalence setting. Subsequent studies performed in a variety of settings confirmed the effectiveness of this regimen, resulting to its adoption as an alternative recommendation for LTBI therapy. ${ }^{57,58}$

Between 2000-2002, a number of reports of increased frequency of side effects emerged, particularly hepatotoxicity requiring discontinuation of therapy. ${ }^{59-61}$ On the basis of these reports, a joint recommendation against the use of this regimen was issued in 2003 by the Centers for Disease Control and Prevention and the American Thoracic Society. ${ }^{62}$ A systematic review of LTBI treatment with 2 month rifampicin and pyrazinamide ultimately concluded that overall, drug discontinuation due to hepatotoxicity occurred in $2.0 \%-17.6 \%$ of HIV-negative patients and $0 \%-9.5 \%$ in patients with HIV co-infection. ${ }^{51}$ Accordingly, this regimen is no longer recommended in international guidelines.

\section{Isoniazid and rifampicin}

Three or four month courses of isoniazid and rifampicin were at least equivalent to 9 months of isoniazid alone in one randomized controlled trial in children $<15$ years old. ${ }^{63}$ In this study, 926 children diagnosed with LTBI were randomized to either a short course or standard isoniazid regimen, and followed for a minimum of 3 years post-treatment. Greater compliance (78\%-89\% vs $65.5 \%$ ) was seen in children prescribed short course regimens.

Subjects who received short course therapy were also found to have less radiologic change suggesting active disease during follow up ( $11 \%$ vs $24 \%$ ); however, no child from either group experienced a clinically and microbiologically diagnosed episode of active tuberculosis. $6 \%$ of children who received isoniazid therapy developed transient increases in liver enzymes, which was seen in $1.2 \%$ of short-course recipients. No patient from either group experienced severe hepatotoxicity or required treatment cessation for adverse effects.

\section{Isoniazid and rifapentine}

The combination of isoniazid and rifapentine has been of recent interest in the treatment of LTBI. Rifapentine is a long-acting rifamycin that has been used successfully as a weekly dose in the continuation phase of treatment for active TB, and has been shown in animal models to be effective against latent TB. ${ }^{64-66}$ Reviews of its use in the treatment of active tuberculosis suggest that it is generally well-tolerated in this setting, and associated with low rates of serious adverse effects. ${ }^{67}$

Weekly isoniazid (900 $\mathrm{mg}$ ) and rifapentine $(900 \mathrm{mg})$ for 12 weeks has been compared to daily rifampicin and pyrazinamide in household contacts of pulmonary TB. ${ }^{68}$ Contacts treated with weekly dosing were less likely to develop hepatotoxicity ( $1 \%$ vs $10 \%$ ). However, this regimen may have been slightly less effective, with active TB infections occurring at a rate of 0.5 per 100 patient-years in the weekly dosing regimen versus 0.2 in the daily rifampicin and pyrazinamide group. This difference was not statistically significant, and both groups experienced substantially less active disease than expected from local background rates of $4 \%$ annually. ${ }^{69}$ The combination of rifapentine and moxifloxacin has not been evaluated in humans, however it demonstrated equal efficacy with rifapentine and isoniazid in a mouse model of LTBI. ${ }^{70}$

Rifapentine appears to be a well-tolerated medication, with potential for shortening courses of LTBI therapy. Further investigation is required into the comparative effectiveness of intermittent rifapentine-containing regimens, and the potential for development of multidrug resistance during therapy.

\section{Treatment of suspected multidrug- resistant latent tuberculosis}

As reviewed above, the considerable bulk of therapies investigated for the treatment of latent TB infection are intended for infections with isolates sensitive to first-line tuberculosis medications. However, with an increasing global burden of multidrug-resistant tuberculosis (MDR TB), the presumption of infection caused by drug-sensitive TB isolates may not be justified in some clinical settings. Most commonly, this relates to people known to have been significantly exposed to a source of sputum smear-positive MDR TB. However, where patients are epidemiologically likely to have acquired LTBI in settings with very high prevalence of MDR TB it may also be questioned whether treatment of latent infection should include the possibility of multidrug-resistance de novo. It has been suggested that latent MDR TB may be less likely to reactivate than infection caused by drugsusceptible organisms, a finding supported by notification data in at least one national surveillance program. ${ }^{71,72}$ It is presently difficult to quantify the likelihood of reactivation MDR TB, however, and the consequences of developing active infection are significant.

Evidence for treatment of LTBI in such settings is limited. A systematic review of this area identified two 
nonrandomized studies considering the effectiveness of LTBI treatment in contacts of known MDR TB index cases. ${ }^{73}$ One prospective study individualized medications included in the treatment regimen based on the sensitivity testing results from the household contact. Medications prescribed included high-dose isoniazid (15-20 mg/kg), ethambutol, ofloxacin and ethionamide. From 105 children with household contact, 41 received individualized therapy. During a 30 -month follow-up period, 2 of 41 (5\%) developed active TB infection, compared with 13 of the $64(20 \%)$ who had not received chemoprophylaxis. A further study retrospectively evaluated the impact of high-dose isoniazid preventive therapy on close contacts of index patients with MDR TB. ${ }^{69}$ A 6-month course of $400 \mathrm{mg}$ /day of isoniazid was initiated for 45 contacts of index patients with tuberculosis, with two contacts subsequently developing active TB. Both of the breakthrough contacts had MDR-TB, as did their index cases. More recently, individualized regimens for treating LTBI have also been used by the US Centers for Disease Control in the setting of MDR-TB outbreaks in Micronesia, although little data has yet been reported regarding effectiveness. ${ }^{74}$ By contrast, on the basis of limited available evidence, the WHO does not recommend the use of second-line agents for treating LTBI. ${ }^{26}$

Even in settings where rates of active MDR TB are extremely high, such as countries of the former Soviet Union, it is unlikely that the routine use of second-line antituberculosis medications for LTBI will be justifiable given the risks of subsequent re-infection and contributing to further drug resistance. However, individualized treatment regimens may be considered in patients based on sensitivity testing in contact isolates and personal risk/benefit considerations.

\section{Cost-effectiveness}

The relative cost-effectiveness of various regimens for treatment of LTBI has been evaluated in several recent studies. Ziakas and Mylonakis compared nine months of daily isoniazid with 4 months of daily rifampicin using meta-analysis of 3586 published patients in four head-to-head trials. ${ }^{75}$ They conclude that rifampicin therapy is associated with significantly decreased discontinuation rates (RR $0.53 ; 95 \% \mathrm{CI}$ : 0.44-0.63), hepatotoxicity (RR 0.12; 95\% CI: 0.05-0.3) and decreased cost (USD 972.20 vs 1062.50) than 9 months of isoniazid. However, the difference in cost in this analysis was heavily influenced by the use of laboratory testing, which assumed monthly testing of liver function and complete blood counts in all patients. Such testing is arguably unnecessary in low risk patients with normal baseline investigation, and may result in unwarranted discontinuation of therapy due to mild abnormalities. ${ }^{76}$ Due to higher cost of rifampicin medication, cost-effectiveness comparison would favor isoniazid if monthly testing were not performed. Nonetheless, frequent laboratory testing is performed in many countries, and this analysis suggests the superior cost-effectiveness of rifampicin under such conditions. A second analysis of cost-effectiveness used a mathematical model that included broader societal and health care costs in the analysis, and also suggested that rifampicin was more cost-effective than isoniazid based regimens. ${ }^{77}$ Both studies highlight the benefits associated with shorter courses of effective LTBI therapy, and suggest that improved completion rates are likely to offset the higher medication cost of rifampicin.

\section{Novel and emerging therapies}

Currently, a number of novel pharmacologic agents with activity against $M$. tuberculosis are in various phases of development. ${ }^{67}$ Several of these medications have begun preliminary assessment in animal models of LTBI, including moxifloxacin and PA-824(70). Although most novel agents have not been directly assessed in the treatment of LTBI, a larger spectrum of available agents will provide greater options for tuberculous therapy and may yield new possibilities for LTBI treatment.

Ultimately, it would be advantageous if LTBI could be treated with a different class of drug from those used for active tuberculosis, reducing concerns regarding the uncertainty of drug resistance patterns in patients with LTBI, as well as limiting the possibility of resistant active TB following failed therapy.

\section{Non-pharmacological therapies}

Finally, the future potential for non-drug treatments for eradication of LTBI, particularly vaccines and other immunotherapies, remains to be fully explored. Vaccines against tuberculosis have been most often conceived as tools for preventing establishment of latent disease or the subsequent emergence of active infection, however more recently there has been interest in the development of vaccines that augment eradication of LTBI. ${ }^{78,79}$ The existing BCG vaccine has a moderate capacity for reducing active infection rates, but no role in post-exposure management of LTBI. ${ }^{80-82}$ However, several novel DNA and subunit vaccines have demonstrated some promise in animal models. One study in a murine LTBI model found that therapeutic DNA vaccination had no effect on reactivation, but when used in conjunction with moxifloxacin treatment augmented its bactericidal effects. ${ }^{70}$ An alternative 
therapeutic vaccine, RUTI, involving liposomally delivered fragmented $M$. tuberculosis cells, has also been shown to enhance the effect of short-course chemotherapy in animal models, with ongoing clinical trials continuing. ${ }^{83,84}$ Effective vaccines against the latent phase of tuberculosis infection would be a welcome adjunct to LTBI management but remain distant from clinical use at present.

Non-vaccine immunotherapy has perhaps been considered more frequently in non-tuberculous mycobacterial infections, however may be of benefit in TB also, particularly in the setting of extensively drug-resistant infection. ${ }^{85}$ Investigations into intravenous immunoglobulin (IVIg) and 16 $\alpha$-bromoepiandrosterone (HE2000) have shown antituberculosis effect, while adjunctive therapy with thalidomide analogue CC-3052 enhances mycobacterial clearance. ${ }^{85-88}$ One small study suggested that adjunctive IL-2 may reduce the duration of smear-positivity during treatment of pulmonary $\mathrm{TB}$, however a subsequent RCT did not confirm this finding. ${ }^{88,89}$ GM-CSF increases phagocytosis of non-tuberculous mycobacteria, and has also been shown to reduce mycobacterial persistence in a murine lung model. ${ }^{90,91}$ Overall, trials of immunotherapy have so far largely concentrated on animal models of active infection however they may have a future role in shortening LTBI treatment regimens when used as adjunctive therapy.

\section{Summary}

Current LTBI therapy is limited by long courses of treatment with concomitant poor adherence, side effects, and uncertain effectiveness in the individual patient. Moreover, the effectiveness of strategies for treating latent tuberculosis may be potentially threatened in many areas by rising rates of drug resistance. To optimize LTBI management in the future, effective short course therapies must be further investigated and instituted, particularly with randomized studies comparing 9 months of isoniazid therapy against alternative regimens.

Further research and clinical experience in the management of MDR-LTBI is imperative, and will become increasingly important in the future. As rates of active tuberculosis infection fall worldwide, appropriate diagnosis and management of LTBI become increasingly important, particularly in countries that have already achieved low prevalence of active disease.

Finally, refinement in epidemiologic approaches to understanding LTBI prevalence and reactivation will be important for translating individual treatment programs into the planning and assessment of effective public health strategies.

\section{Disclosure}

The authors report no conflicts of interest in this work.

\section{References}

1. World Health Organization. Global tuberculosis control: a short update to the 2009 report. 2009. Available from: http://www.who.int/tb/publications/global_report/2009/update/en/. Accessed March 1, 2010.

2. Tufariello J, Chan J, Flynn J. Latent tuberculosis: mechanisms of host and bacillus that contribute to persistent infection. Lancet Infect Dis. 2003;3(9):578-590.

3. Cohn D, O'Brien R. Targeted tuberculin testing and treatment of latent tuberculosis infection. MMWR Morb Mortal Wkly Rep. 2000;4(9): $1-61$.

4. White M, Duong T, Cruz E, et al. Strategies for effective education in a jail setting: the Tuberculosis Prevention Project. Health Promotion Practice. 2003;4(4):422.

5. Tavitian S, Spalek V, Bailey R. A pharmacist-managed clinic for treatment of latent tuberculosis infection in health care workers. Am J Health-Sys Pharm. 2003;60(18):1856-1861.

6. Hill L, Blumberg E, Sipan C, et al. Multi-Level Barriers to LTBI Treatment: A Research Note. J Immig Minority Health. 2008:1-7.

7. An Interim Report of the Medical Research Council by their Tuberculosis Chemotherapy Clinical Trials Committee. The treatment of pulmonary tuberculosis with isoniazid. BMJ. 1952;2:735-746.

8. Corbett E, Watt C, Walker N, et al. The growing burden of tuberculosis: global trends and interactions with the HIV epidemic. Arch Internal Med. 2003;163(9):1009.

9. Narain J, Raviglione M, Kochi A. HIV-associated tuberculosis in developing countries: epidemiology and strategies for prevention. Tuberc Lung Dis. 1992;73(6):311-321.

10. Manosuthi W, Chottanapand S, Thongyen S, et al. Survival rate and risk factors of mortality among HIV/tuberculosis-coinfected patients with and without antiretroviral therapy. JAIDS. 2006;43(1):42.

11. Swaminathan S, Paramasivan C, Kumar S, et al. Unrecognized tuberculosis in HIV-infected patients: sputum culture is a useful tool. Int $J$ TB Lung Dis. 2004;8(7):896-898.

12. Danel C, Ouassa T, Moh R, et al. Screening for active tuberculosis before INH chemoprophylaxis in West African adults with high CD4 counts: inclusion phase of temprano ANRS 12136. CROI. 2010 Feb 16-19; Montreal, Canada.

13. Bernstein J, Lott W, Steinberg B, Yale H. Chemotherapy of experimental tuberculosis. V. Isonicotinic acid hydrazide (nydrazid) and related compounds. Am Rev Tuberc. 1952;65(4):357.

14. Takayama K, Wang L, David H. Effect of isoniazid on the in vivo mycolic acid synthesis, cell growth, and viability of Mycobacterium tuberculosis. Antimicrobial Agents and Chemotherapy. 1972;2(1):29.

15. Egsmose T, Ang'awa JO, Poti SJ. The use of isoniazid among household contacts of open cases of pulmonary tuberculosis. Bull WHO. 1965; 33:419-433.

16. Ewer K, Millington KA, Deeks JJ, et al. Dynamic antigen-specific T-cell responses after point-source exposure to Mycobacterium tuberculosis. Am J Resp Crit Care Med. 2006;174:831-839.

17. Thompson N. Efficacy of various durations of isoniazid preventive therapy for tuberculosis: five years of follow-up in the IUAT trial. International Union Against Tuberculosis Committee on Prophylaxis. Bull WHO. 1982;60(4):555-564.

18. Comstock G. How much isoniazid is needed for prevention of tuberculosis among immunocompetent adults? Counterpoint. Int J TB Lung Dis. 1999;3(10):847-850.

19. Zar H, Cotton M, Strauss S, et al. Effect of isoniazid prophylaxis on mortality and incidence of tuberculosis in children with HIV: randomised controlled trial. BMJ. 2007;334(7585):136.

20. le Roux S, Cotton M, Golub J, et al. Adherence to isoniazid prophylaxis among HIV-infected children: a randomized controlled trial comparing two dosing schedules. BMC Med. 2009;7(1):67. 
21. Bucher H, Griffith L, Guyatt G, et al. Isoniazid prophylaxis for tuberculosis in HIV infection: a meta-analysis of randomized controlled trials. AIDS. 1999;13(4):501-507.

22. Grant A, Charalambous S, Fielding K, et al. Effect of routine isoniazid preventive therapy on tuberculosis incidence among HIV-infected men in South Africa: a novel randomized incremental recruitment study. JAMA. 2005;293(22):2719.

23. Nguyen T, Odermatt P, Slesak G, Barennes H. Risk of latent tuberculosis infection in children living in households with tuberculosis patients a cross sectional survey in remote northern Lao People's Democratic Republic. BMC Infect Dis. 2009;9(1):96.

24. Morrison J, Pai M, Hopewell P. Tuberculosis and latent tuberculosis infection in close contacts of people with pulmonary tuberculosis in low-income and middle-income countries: a systematic review and meta-analysis. Lancet Infect Dis. 2008;8(6):359-368.

25. Mount F, Ferebee S. Preventive effects of isoniazid in the treatment of primary tuberculosis in children. New Eng J Med. 1961;265:713-721.

26. World Health Organisation. Guidance for national tuberculosis programmes on the management of tuberculosis in children. 2006 Available from http://www.who.int/child_adolescent_health/documents/ htm_tb_2006_371/en/index.html

27. Zachariah R, Spielmann M, Harries A, et al. Passive versus active tuberculosis case finding and isoniazid preventive therapy among household contacts in a rural district of Malawi. Int J TB Lung Dis. 2003;7(11): 1033-1039.

28. Carter E, Mates S. Tuberculosis during pregnancy. The Rhode Island experience, 1987 to 1991. Chest. 1994;106(5):1466-1470.

29. Scheinhorn D, Angelillo V. Antituberculous therapy in pregnancy: risks to the fetus. West J Med. 1977;127(3):195-198.

30. Franks A, Binkin N, Snider D Jr, et al. Isoniazid hepatitis among pregnant and postpartum Hispanic patients. Pub Health Rep. 1989;104(2):151.

31. Millard P, Wilcosky T, Reade-Christopher S, Weber D. Isoniazid-related fatal hepatitis. West J Med. 1996;164(6):486-491.

32. Ormerod P. Respiratory diseases in pregnancy. 3: Tuberculosis in pregnancy and the puerperium. BMJ. 2001;56(6):494-499.

33. Boggess K, Myers E, Hamilton C. Antepartum or postpartum isoniazid treatment of latent tuberculosis infection. Obstet Gyn. 2000;96(5 Pt 1): 757-762.

34. Snider Jr D, Powell K. Should women taking anti-tuberculosis drugs breast-feed? Arch Int Med. 1984;144(3):589.

35. Fountain F, Tolley E, Jacobs A, Self T. Rifampin hepatotoxicity associated with treatment of latent tuberculosis infection. Am J Med Sci. 2009;337(5):317-320.

36. Nolan C, Goldberg S, Buskin S. Hepatotoxicity associated with isoniazid preventive therapy: a 7-year survey from a public health tuberculosis clinic. JAMA. 1999;281(11):1014-1018.

37. Saukkonen J, Cohn D, Jasmer R, et al. An official ATS statement: hepatotoxicity of anti-tuberculosis therapy. Am J Resp Crit Care Med. 2006;174(8):935-952.

38. Byrd R, Horn B, Solomon D, Griggs G. Toxic effects of isoniazid in tuberculosis chemoprophylaxis. Role of biochemical monitoring in 1,000 patients. JAMA. 1979;241(12):1239-1241.

39. Stuart R, Wilson J, Grayson ML. Isoniazid toxicity in health care workers. Clin Infect Dis. 1999;28(4):895-897.

40. Menzies D, Long R, Trajman A, et al. Adverse events with 4 months of rifampin therapy or 9 months of isoniazid therapy for latent tuberculosis infection: a randomized trial. Ann Intern Med. 2008;149(10):689-697.

41. Brown M, Howard E, DeAlleaume L. Are liver function tests required for patients taking isoniazid for latent TB? J Family Pract. 2004;53(1):63-67.

42. Devadatta S, Gangadharam P, Andrews R, et al. Peripheral neuritis due to isoniazid. Bull WHO. 1960;23:587-598.

43. Meyer U. Pharmacogenetics and adverse drug reactions. Lancet. 2000; 356(9242):1667-1671.

44. Denholm J, Wesselingh S. Chapter 135: Stavudine. In: Grayson ML, Mills J, editors. Kucer's The Use of Antibiotics. Melbourne: Hodder Arnold; 2010.
45. Marais B, Graham S, Cotton M, Beyers N. Diagnostic and management challenges for childhood tuberculosis in the era of HIV. J Infect Dis. 2007;196(S1):76-85.

46. Schaaf H, Marais B, Hesseling A, et al. Childhood drug-resistant tuberculosis in the Western Cape Province of South Africa. Acta Paediatrica. 2006;95(5):523-528.

47. Wright A, Zignol M, Van Deun A, et al. Epidemiology of anti-tuberculosis drug resistance 2002-2007: an updated analysis of the Global Project on Anti-Tuberculosis Drug Resistance Surveillance. Lancet. 2009; 368(9553):2142-2154.

48. Girling D, Chan S. A double-blind placebo-controlled clinical trial of three anti-tuberculosis chemoprophylaxis regimens in patients with silicosis in Hong Kong. Am Rev Resp Dis. 1992;145:36-41.

49. Polesky A, Farber H, Gottlieb D, et al. Rifampin preventive therapy for tuberculosis in Boston's homeless. Am J Resp Crit Care Med. 1996; 154(5):1473-1477.

50. Reichman L, Lardizabal A, Hayden C. Considering the role of four months of rifampin in the treatment of latent tuberculosis infection. Am J Resp Crit Care Med. 2004;170(8):832-835.

51. Sterling T. New approaches to the treatment of latent tuberculosis. Semin Respir Crit Care Med. 2008;29:532-541.

52. Burman W, Jones B. Treatment of HIV-related tuberculosis in the era of effective antiretroviral therapy. Am J Resp Crit Care Med. 2001; 164(1):7-12.

53. Moreno S, Podzamczer D, Blázquez R, et al. Treatment of tuberculosis in HIV-infected patients: safety and antiretroviral efficacy of the concomitant use of ritonavir and rifampin. AIDS. 2001;15(9): 1185-1187.

54. Villarino M, Ridzon R, Weismuller $\mathrm{P}$, et al. Rifampin preventive therapy for tuberculosis infection: experience with 157 adolescents. Am J Resp Crit Care Med. 1997;155(5):1735-1738.

55. Mitrano J, Spooner L, Belliveau P. Excretion of antimicrobials used to treat methicillin-resistant Staphylococcus aureus infections during lactation: safety in breastfeeding infants. Pharmacotherapy. 2009;29(9): 1103-1109.

56. Mwinga A, Hosp M, Godfrey-Faussett P, et al. Twice weekly tuberculosis preventive therapy in HIV infection in Zambia. AIDS. 1998;12(18):2447-2457.

57. Halsey N, Coberly J, Desormeaux J, et al. Randomised trial of isoniazid versus rifampicin and pyrazinamide for prevention of tuberculosis in HIV-1 infection. Lancet. 1998;351(9105):786-792.

58. Gordin F, Chaisson R, Matts J, et al. An international, randomized trial of rifampin and pyrazinamide versus isoniazid for prevention of tuberculosis in HIV-infected persons. JAMA. 2000;283:1445-1450.

59. Centers for Disease Control and Prevention. Fatal severe hepatitis associated with rifampin and pyrazinamide for the treatment of latent tuberculosis infection - New York and Georgia, 2000. MMWR. 2001;50: 289-291.

60. Centers for Disease Control and Prevention. Update: fatal and severe liver injuries associated with rifampin and pyrazinamide for latent tuberculosis infection, and revisions in American Thoracic Society/ CDC recommendations - United States, 2001. MMWR. 2001;50: $733-735$.

61. Centers for Disease Control and Prevention. Update: fatal and severe liver injuries associated with rifampin and pyrazinamide treatment for latent tuberculosis infection. MMWR Morb Mortal Wkly Rep. 2002;51:998-999.

62. Centers for Disease Control and Prevention. Update: adverse event data and revised American Thoracic Society/CDC recommendations against the use of rifampin and pyrazinamide for treatment of latent tuberculosis infection - United States, 2003. MMWR Morb Mortal Wkly Rep. 2003;52:735-739.

63. Spyridis N, Spyridis P, Gelesme A, et al. The effectiveness of a 9-month regimen of isoniazid alone versus 3-and 4-month regimens of isoniazid plus rifampin for treatment of latent tuberculosis infection in children: results of an 11-year randomized study. Clin Infect Dis. 2007;45(6): $715-722$. 
64. Benator D, Bhattacharya M, Bozeman L, et al. Rifapentine and isoniazid once a week versus rifampicin and isoniazid twice a week for treatment of drug-susceptible pulmonary tuberculosis in HIV-negative patients: a randomised clinical trial. Lancet. 2002;360(9332):528-535.

65. Burman W, Gallicano K, Peloquin C. Comparative pharmacokinetics and pharmacodynamics of the rifamycin antibacterials. Clin Pharmacokinetics. 2001;40(5):327-341.

66. Ji B, Truffot-Pernot C, Lacroix C, et al. Effectiveness of rifampin, rifabutin, and rifapentine for preventive therapy of tuberculosis in mice. Am Rev Resp Dis. 1993;148(6 Pt 1):1541-1546.

67. van den Boogaard J, Kibiki G, Kisanga E, et al. New drugs against tuberculosis: problems, progress, and evaluation of agents in clinical development. Antimicrobial Agents Chemotherapy. 2009;53(3):849-862.

68. Schechter M, Zajdenverg R, Falco G. et al. Weekly rifapentine/isoniazid or daily rifampin/pyrazinamide for latent tuberculosis in household contacts. Am J Resp Crit Care Med, 2006;173(8):922-926.

69. Kritski A, Marques M, Rabahi M, et al. Transmission of tuberculosis to close contacts of patients with multidrug-resistant tuberculosis. Am J Resp Crit Care Med. 1996;153(1):331-335.

70. Nuermberger E, Tyagi S, Williams K, et al. Rifapentine, moxifloxacin, or DNA vaccine improves treatment of latent tuberculosis in a mouse model. Am J Resp Crit Care Med. 2005;172(11):1452-1456.

71. Attamna A, Chemtob D, Attamna S, et al. Risk of tuberculosis in close contacts of patients with multidrug resistant tuberculosis: a nationwide cohort. Thorax. 2009 Mar 1 2009;64(3):271.

72. Burgos M, DeRiemer K, Small P, et al. Effect of drug resistance on the generation of secondary cases of tuberculosis. J Infect Dis. 2003;188(12):1878-1884.

73. Fraser A, Paul M, Attamna A, Leibovici L. Treatment of latent tuberculosis in persons at risk for multidrug-resistant tuberculosis: systematic review. Int J TB Lung Dis. 2006;10(1):19-23.

74. Centers for Disease Control and Prevention. Two simultaneous outbreaks of multidrug-resistant tuberculosis - Federated States of Micronesia, 2007-2009. MMWR. 2009;58(10):253-256.

75. Ziakas P, Mylonakis E. 4 months of rifampin compared with 9 months of isoniazid for the management of latent tuberculosis infection: a meta-analysis and cost-effectiveness study that focuses on compliance and liver toxicity. Clin Infect Dis. 2009;49(12);1883-1889.

76. Brown MO. Are liver function tests required for patients taking isoniazid for latent TB? J Family Prac. 2004;53(1):63-66.

77. Holland D, Sanders G, Hamilton C, Stout J. Costs and cost-effectiveness of four treatment regimens for latent tuberculosis infection. Am J Resp Crit Care Med. 2009;179(11):1055-1060.
78. Andersen P. Vaccine strategies against latent tuberculosis infection. Trends Microbiol. 2007;15(1):7-13.

79. Repique C, Li A, Collins F, Morris S. DNA immunization in a mouse model of latent tuberculosis: effect of DNA vaccination on reactivation of disease and on re-infection with a secondary challenge. Infect Immunity. 2002;70(7):3318-3323.

80. Andersen P. TB vaccines: progress and problems. Trends Immunol. 2001;22(3):160-168.

81. Colditz G, Brewer T, Berkey C. et al. Efficacy of BCG vaccine in the prevention of tuberculosis. Meta-analysis of the published literature. JAMA. 1994;271(9):698-702.

82. Turner J, Rhoades E, Keen M, Belisle J, Frank A, Orme I. Effective pre-exposure tuberculosis vaccines fail to protect when they are given in an immunotherapeutic mode. Infect Immunity. 2000;68(3): 1706-1709.

83. Cardona P. RUTI: a new chance to shorten the treatment of latent tuberculosis infection. Tuberculosis. 2006;86(3-4):273-289.

84. Lambert P, Hawkridge T, Hanekom W. New vaccines against tuberculosis. Clinics Chest Med. 2009;30(4):811-826.

85. Churchyard G, Kaplan G, Fallows D, et al. Advances in immunotherapy for tuberculosis treatment. Clinics Chest Med. 2009;30(4):769-782.

86. Stickney D, Noveljic Z, Garsd A, et al. Safety and activity of the immune modulator HE2000 on the incidence of tuberculosis and other opportunistic infections in AIDS patients. Antimicrobial Agents and Chemotherapy. 2007;51(7):2639-2641.

87. Roy E, Stavropoulos E, Brennan J, et al. Therapeutic efficacy of highdose intravenous immunoglobulin in Mycobacterium tuberculosis infection in mice. Infection immunity. 2005;73(9):6101-6109.

88. Johnson J, Ssekasanvu E, Okwera A, et al. Randomized trial of adjunctive interleukin-2 in adults with pulmonary tuberculosis. Am J Resp Crit Care Med. 2003;168(2):185-191.

89. Johnson B, Bekker L, Rickman R, et al. rhulL-2 adjunctive therapy in multidrug resistant tuberculosis: a comparison of two treatment regimens and placebo. Tuberc Lung Dis. 1997;78(3-4):195-203.

90. Kedzierska K, Mak J, Mijch A, et al. Granulocyte-macrophage colony-stimulating factor augments phagocytosis of Mycobacterium avium complex by human immunodeficiency virus type 1-infected monocytes/macrophages in vitro and in vivo. J Infect Dis. 1999;181(1): 390-394.

91. Szeliga J, Daniel D, Yang C, et al. Granulocyte - macrophage colony stimulating factor-mediated innate responses in tuberculosis. Tuberculosis. 2008;88(1):7-20.
Infection and Drug Resistance

\section{Publish your work in this journal}

Infection and Drug Resistance is an international, peer-reviewed openaccess journal that focuses on the optimal treatment of infection (bacterial, fungal and viral) and the development and institution of preventive strategies to minimize the development and spread of resistance. The journal is specifically concerned with the epidemiology of antibiotic

\section{Dovepress}

resistance and the mechanisms of resistance development and diffusion in both hospitals and the community. The manuscript management system is completely online and includes a very quick and fair peerreview system, which is all easy to use. Visit http://www.dovepress.com/ testimonials.php to read real quotes from published authors. 